\title{
THE MOST PROBABLE HYDROLOGIC RESPONSE OF FRACTAL RIVER NETWORKS
}

\author{
Pierluigi CLAPS, Mauro FIORENTINO and Giuseppe OLIVETO \\ Dipartimento di Ingegneria e Fisica dell'Ambiente \\ Università della Basilicata \\ Via della Tecnica 3 \\ 85100, Potenza (Italy)
}

\begin{abstract}
An expression of the hydrologic response of fractal networks is derived by means of the classical approach used in statistical-mechanics. Probabilities of the rate of arrivals at the outlet of mass particles instantaneously injected into the network are maximized accounting for some suitable constraints. The analysis of the structure of fractal networks allows us to meaningfully set the required constraints, that are of the same form of these used by Lienhard and Meyer (1967). The resulting hydrologic response is a Weibull distribution whose parameters are the fractal dimension and the total length of the network. A comparison with the similar form of arrival time distribution obtained by Troutman and Karlinger (1985) showed it to be a particular case of the more general expression relative to fractal networks.
\end{abstract}

\section{INTRODUCTION}

The study of river network morphology in view of the definition of characters of the basin response characteristics has received new breed since the introduction of fractal geometry concepts in geomorphology. It is now widely accepted that river networks are fractal (i.e. self-similar) objects (e.g. Mandelbrot, 1983; La Barbera and Rosso, 1989) and that their structure obeys some principles of least energy dissipation (e.g. Rinaldo et al., 1992; Fiorentino et al., 1993). Despite that, the concepts of fractal geometry have not yet been extensively used in the evaluation of the basin response. Probably the only attempt in this field is due to Marani et al. (1991) who determined the most probable response of a particular fractal network, the Peano plane-filling curve.

In this paper, a framework for the definition of purely fractal networks is used in the search of one expression of the most probable hydrologic response of river networks. Following the statistical-mechanical approach of Lienhard (1964), structural properties of fractal networks are explicitly used in this search, leading to an IUH form based on clearly recognizable parameters. 


\section{STATISTICAL-MECHANICAL DERIVATION OF THE IUH}

Lienhard (1964), first used a statistical-mechanical approach in the search for a function describing the arrival times of water particles fallen onto the basin, function representing the basin IUH.

The framework used by Lienhard is made up of a drainage-basin system on which $\mathrm{N}$ particles of equal mass are instantaneously injected. These particles fall on regions (states) of the basin with different distances to the outlet. The distances are considered proportional to the arrival times of the water particles to the outlet, so the drainage-basin can be subdivided in a number of time partitions. Using $m$ partitions of time, the compound probability that $\left(\mathrm{N}_{1}\right.$, $\left.\mathrm{N}_{2}, \ldots, \mathrm{N}_{m}\right)$ particles reach the basin outlet respectively at times $\left(\mathrm{t}_{1}, \mathrm{t}_{2}, \ldots, \mathrm{t}_{m}\right)$ is

$$
P\left(N_{l}=n_{l}, \ldots \ldots \ldots, N_{m}=n_{m}\right)=\left(\begin{array}{c}
N \\
{ }_{l}, \ldots \ldots, n_{m}
\end{array}\right) \cdot g_{l}^{n_{l}} \cdot \ldots \ldots \ldots \ldots \cdot g_{m}^{n_{m}}
$$

with $\mathrm{g}_{\mathrm{i}}$ as the number of distinguishable ways of placing $\mathrm{n}_{\mathrm{i}}$ particles in the $i$-th time partition and with $\Sigma \mathrm{N}_{\mathrm{i}}=\mathrm{N}$. Gupta and Waymire (1983), reviewing Lienhard's approach, gave $\mathrm{g}_{\mathrm{i}}$ the interpretation of probabilities $p_{i}$ proportional to the contributing area $C_{i}$ at time $t_{i}=i \Delta t$, so that

$$
\mathrm{p}_{\mathrm{i}}=\mathrm{C}_{\mathrm{i}} / \mathrm{A} \quad 1 \leq \mathrm{i} \leq \mathrm{m}
$$

where $A$ is the basin area. Thus $p_{i}$ represents the probability of having $n_{i}$ particles in the $i$-th contributing area.

The most probable distribution satisfying the above requirements is obtained by maximizing the joint probability (1), for instance by using the principle of maximum entropy (Jaynes, 1957), subject to a set of constraints. In the generalization of Lienhard's approach, Lienhard and Meyer (1967) used the following constraints:

1) a conservation principle:

$$
\sum_{i=1}^{\infty} \mathrm{N}_{\mathrm{i}}=\mathrm{N}
$$

2) a constraint on the moment of order $\beta$ with respect to the origin, considering distance expressed in time units and based on the assumption of uniform velocity throughout the basin:

$$
\mathrm{E}\left[\mathrm{t}^{\beta}\right]=\sum_{i=1}^{\infty} \frac{\mathrm{N}_{\mathrm{i}}}{\mathrm{N}} \cdot \mathrm{t}_{\mathrm{i}}^{\beta}=\mathrm{k} \quad(\mathrm{k}>0)
$$

3) the proportionality between $g_{i}$ and a given power of $t_{i}$ :

$$
\mathrm{g}_{\mathrm{i}}=c \cdot \mathrm{t}_{\mathrm{i}}^{\alpha-1}
$$

with constant $c$ and $\alpha$.

The set $\left(\widetilde{N}_{1}, \widetilde{N}_{2}, \ldots ., \tilde{N}_{i}, \ldots ..\right)$ of particles that allow the maximization of (1) determines a discrete distribution $\mathrm{P}\left(\mathrm{t}_{\mathrm{i}-1} \leq \mathrm{T} \leq \mathrm{t}_{\mathrm{i}-1}+\Delta \mathrm{t}\right)=\widetilde{N}_{i} / \mathrm{N}$, that can be approximated with a probability density function $\mathrm{f}(\mathrm{t})$ according to

$$
\frac{\tilde{N} i}{N}=\int_{t_{i-1}}^{t_{i-1}+\Delta t} f(t) d t
$$


Letting $\Delta$ t tend to zero, the $p d f$ that maximizes (1) is a generalized Gamma distribution (Lienhard and Meyer, 1967)

$$
f(t)=\left[\frac{\beta}{\Gamma(\alpha / \beta)} \cdot\left(\frac{\alpha}{\beta k}\right)^{\alpha / \beta}\right] \cdot t^{\alpha-1} \cdot \exp \left[-\frac{\alpha t^{\beta}}{\beta k}\right] \quad t \geq 0
$$

characterized by the three parameters $\alpha, \beta$ and $\mathrm{k}$.

In the scheme introduced by Lienhard (1964) parameters are set as $\alpha=n+1$ and $\beta=2$. For $\beta=1$ the distribution (7) is reduced to a two-parameter Gamma while for $\alpha=\beta$ (7) becomes a Weibull distribution:

$$
f(t)=\frac{\alpha}{k} t^{\alpha-1} \exp \left(-\frac{t^{\alpha}}{k}\right)
$$

In the search for the most probable $p d f$ of arrival times, constraints play the role of conditions that lead to incorporating some peculiar and clearly recognizable characteristics of the drainage-network system into the $p d f$ expression. In fact, this is not the case of the approaches by Lienhard (1964) and Lienhard and Meyer (1967), as recognized by Marani et al. (1991) who dropped the dynamic constraint due to its arbitrary definition.

The main purpose of this paper is to show how a fractal framework, established with a set of properties of the objects, can allow us to impose some meaningful constraints in the maximization of a joint probability expression, leading to a result analogous to (7).

A fundamental point in this approach is that we consider uniquely the response due to the channel network. No hillslope or area effects are taken into account. This hypothesis is not too hard, if one considers the role of the geomorphological factor in the whole response of the basin (e.g. Rinaldo et al., 1991). Moreover, it is to be considered that only the topological structure of the network is taken into account, which considers segments of equal length and is functional to the application of the rules of construction of fractal networks.

Characteristics and properties of the fractal objects used in this analysis are introduced in the next section.

\section{A FRAMEWORK FOR THE DEFINITION OF FRACTAL NETWORKS}

Fractal networks are built with the very simple rules of construction of self-similar objects, using a an initiator, which is a unit-length segment, and a generator, which is a tree-type combination of equal shorter segments (Mandelbrot, 1983, pp.72-73) whose length is $\eta$. Segments of the tree are of equal length are straight and form only square angles, so that the longest path in the generator tree (called topological diameter $\Delta_{1}$ ) is always the initiator and is made up of $1 / \eta$ segments.

After the first substitution of the initiator with the generator, each segment of the generator becomes an initiator and is substituted again (figure 1), in a recursive way (e.g. Feder, 1988, p. 16). After $m$ multiplications, the number of segments is $\mathrm{M}=\mathrm{N}_{1}{ }^{m}$, where $\mathrm{N}_{1}$ is the number of segments of the generator, and the segment length is $\zeta_{m}=\eta^{m}$. 
In the network obtained after $m$ multiplications the topological diameter $\Delta_{\mathrm{m}}$ is equal to (Claps and Oliveto, 1993)

$$
\Delta_{\mathrm{m}}=\Delta_{1}^{\mathrm{m}}
$$

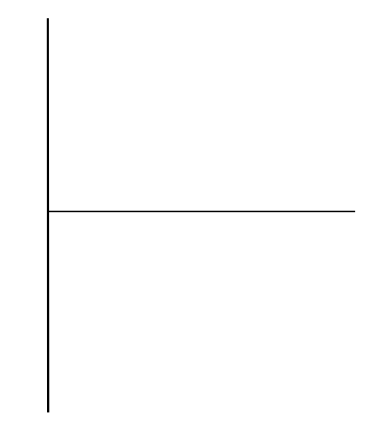

$m=1$

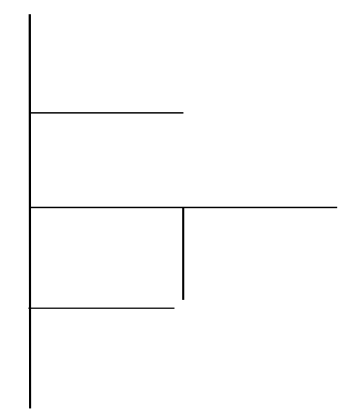

$m=2$

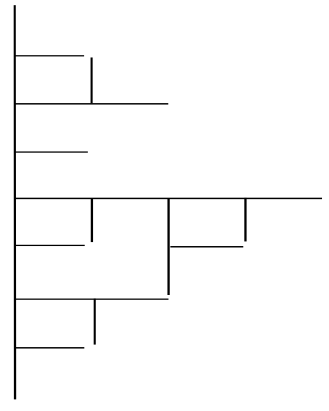

$m=3$

Fig. 1. Generation of a fractal tree. Parameter $m$ is the generation index. The structure with $m=1$ is the generator. The initiator is a simple segment for all the cases considered.

The fractal dimension of these geometric sets is defined as (e.g. Feder, 1988, p.19)

$$
\mathrm{D}_{\mathrm{t}}=-\frac{\ln N_{1}}{\ln \eta}
$$

Since we are dealing only with straight segments, this fractal dimension is to be considered as due only to the branching process governing network growth (is a topological fractal dimension). Coefficient $\mathrm{D}_{\mathrm{t}}$ can also be called the network similarity dimension.

\section{1. determination of contributing areas for fractal networks}

Mandelbrot (1983) first suggested that basin area should be proportional to the fractal measure of the total network length. This hypothesis was adopted by Marani et al. (1991) as a connectivity conjecture implying constant drainage density. In this paper this hypothesis is extended in considering the basin area proportional to the fractal measure of the length $\mathrm{Z}$ of the network. The fractal (invariant) measure $\Lambda$ of a self-similar object is (Mandelbrot, 1983)

$$
\Lambda=\mathrm{N}_{\varepsilon} \varepsilon^{\mathrm{D}}
$$

where $\varepsilon$ is the ruler length, $\mathrm{N}_{\varepsilon}$ is the number of rulers needed to cover the object and D is the fractal dimension. In the case discussed, the fractal measure $\mathrm{Z}_{\mathrm{i}}$ of the complete topological length of a subnetwork is

$$
\mathrm{Z}_{\mathrm{i}}=\mathrm{M}_{\mathrm{i}} \zeta^{\mathrm{D}_{\mathrm{t}}}
$$

with $\mathrm{M}_{\mathrm{i}}$ as the number of links of the subnetwork, $\zeta$ as the link length and $\mathrm{D}_{\mathrm{t}}$ as the similarity dimension of the fractal network. The index $m$ of generation is dropped here and in the following, without loss of generality. 
To define contributing areas $\mathrm{C}_{\mathrm{i}}$ Gupta and Waymire (1983) made reference to the schematic basin depicted in figure 2 and used cumulative areas $A_{i}$ as follows:

$$
\mathrm{C}_{\mathrm{i}}=\frac{d \mathrm{~A}_{\mathrm{i}}}{d \mathrm{~L}_{\mathrm{i}}} d \mathrm{~L}_{\mathrm{i}}
$$

Based on the previous hypothesis, to reformulate (13) for fractal networks the cumulative area $A_{i}$ is substituted by the total length $Z_{i}$ of the subnetwork and the distance $L_{i}$ of the contributing area from the outlet is assumed as the length of the mainstream of the corresponding subnetwork.

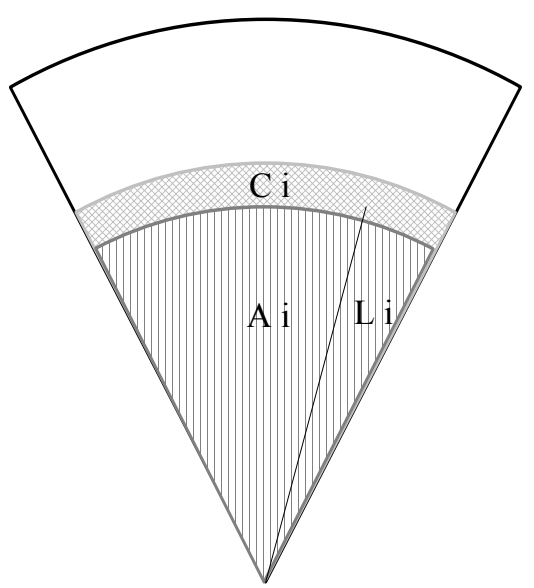

Fig. 2. Representation of a basin shaped as a sector of circle (from Gupta and Waymire, 1983).

One can thus write (13) as the derivative of $Z_{i}$ with respect to the subnetwork mainstream length

$$
\mathrm{C}_{\mathrm{i}}=\frac{\mathrm{dZ}_{\mathrm{i}}}{\mathrm{dL}_{\mathrm{i}}} \mathrm{dL}_{\mathrm{i}}
$$

Since in fractal networks $\mathrm{L}_{\mathrm{i}}=\Delta_{\mathrm{i}} \zeta$, with $\Delta_{\mathrm{i}}$ as the subnetwork topological diameter, and given $\Delta_{\mathrm{i}}$ as a discrete integer quantity, so that $\mathrm{d} \Delta_{\mathrm{i}}=1, \mathrm{dL}_{\mathrm{i}}=\zeta$ and relation (14) becomes

$$
C_{i}=\zeta^{D_{t}} \frac{d_{i}}{d L_{i}} d L_{i}=\zeta^{D_{t}} \frac{d_{i}}{d \Delta_{i}}
$$

Claps and Oliveto (1993) found for fractal networks the following relation between total number of links and topological diameter:

$$
\mathrm{M}_{\mathrm{i}}=\Delta_{\mathrm{i}}^{\mathrm{D}_{\mathrm{t}}}
$$

holding strictly for subnetworks obtainable with repeated substitutions of the generator (called m-structures in Claps and Oliveto, 1993) but resulting in a very good relation for all others subnetworks, as can be seen in figure 3. In relation (15) and in the following, the network topological diameter is indicated by $\Delta$, with no mention to the (generic) index $m$ of generation; thus $\Delta_{\mathrm{i}}$ in (15) denotes the diameter of a subnetwork $i$ as a part of a larger 
network.
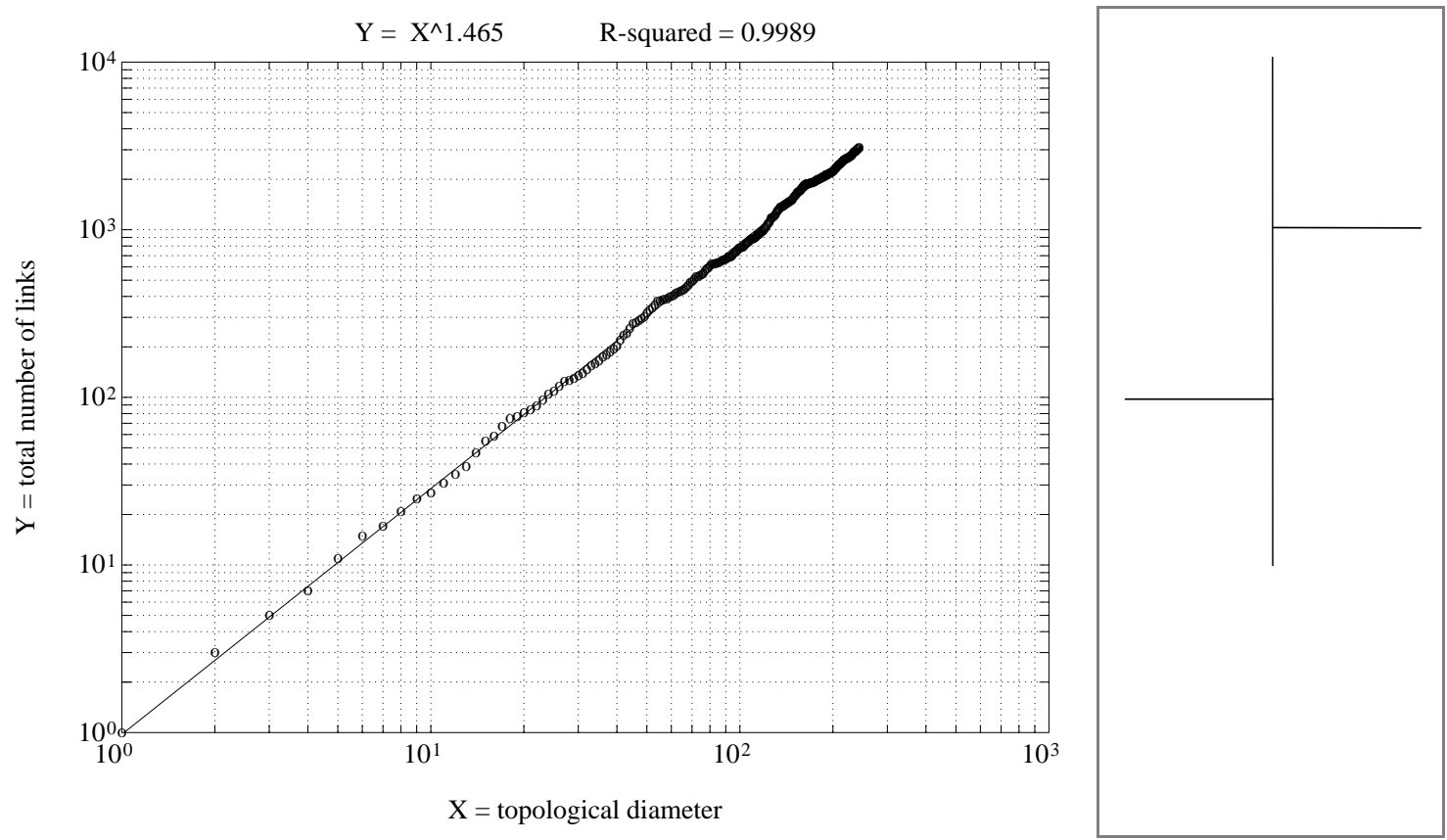

Fig. 3. Relation between magnitude and topological diameter of all subnetworks of a fractal network generated from the elementary tree represented in the rectangle on the right. The fractal dimension of the tree is $D t=1.465$.

Deriving relation (16) one obtains

$$
\frac{\mathrm{dM}_{\mathrm{i}}}{\mathrm{d} \Delta_{\mathrm{i}}}=\mathrm{D}_{\mathrm{t}} \cdot \Delta_{\mathrm{i}}^{\mathrm{D}_{\mathrm{t}}-1}
$$

so that (15) can be rewritten as

$$
\mathrm{C}_{\mathrm{i}}=\zeta^{\mathrm{D}_{\mathrm{t}}} \cdot \mathrm{D}_{\mathrm{t}} \Delta_{\mathrm{i}}^{\mathrm{D}_{\mathrm{t}}-1}
$$

Using $\mathrm{L}_{\mathrm{i}}$ (expressed as $\Delta_{\mathrm{i}} \zeta$ ) for a meaningful comparison with the constraint (5), the condition imposed on (19), given constant velocity, $C_{i}$ by the fractal nature of the network is

$$
\mathrm{C}_{\mathrm{i}}=\zeta \mathrm{D}_{\mathrm{t}} \mathrm{L}_{\mathrm{i}}^{\mathrm{D}_{\mathrm{t}}-1}
$$

that leads to probabilities $\mathrm{p}_{\mathrm{i}}$ (equivalent to $\mathrm{g}_{\mathrm{i}}$ ):

$$
\mathrm{p}_{\mathrm{i}}=\frac{\mathrm{C}_{\mathrm{i}}}{\mathrm{Z}}=\frac{\mathrm{D}_{\mathrm{t}} \cdot \zeta \cdot \mathrm{L}_{\mathrm{i}}^{\mathrm{D}_{\mathrm{t}}-1}}{\mathrm{Z}}
$$

where the constant $c$ of (5) is represented by the ratio

$$
c=\frac{\mathrm{D}_{\mathrm{t}} \zeta_{\mathrm{v}} \mathrm{D}_{\mathrm{t}}-1}{\mathrm{Z}}
$$

with $\mathrm{v}$ as the velocity. 
Relation (20) is shown in figure 4, as compared to the topological width function, representing the number of links at a given with reference to the same fractal tree used in figure 3 , at the same distance from the outlet normalized by the total number of links.
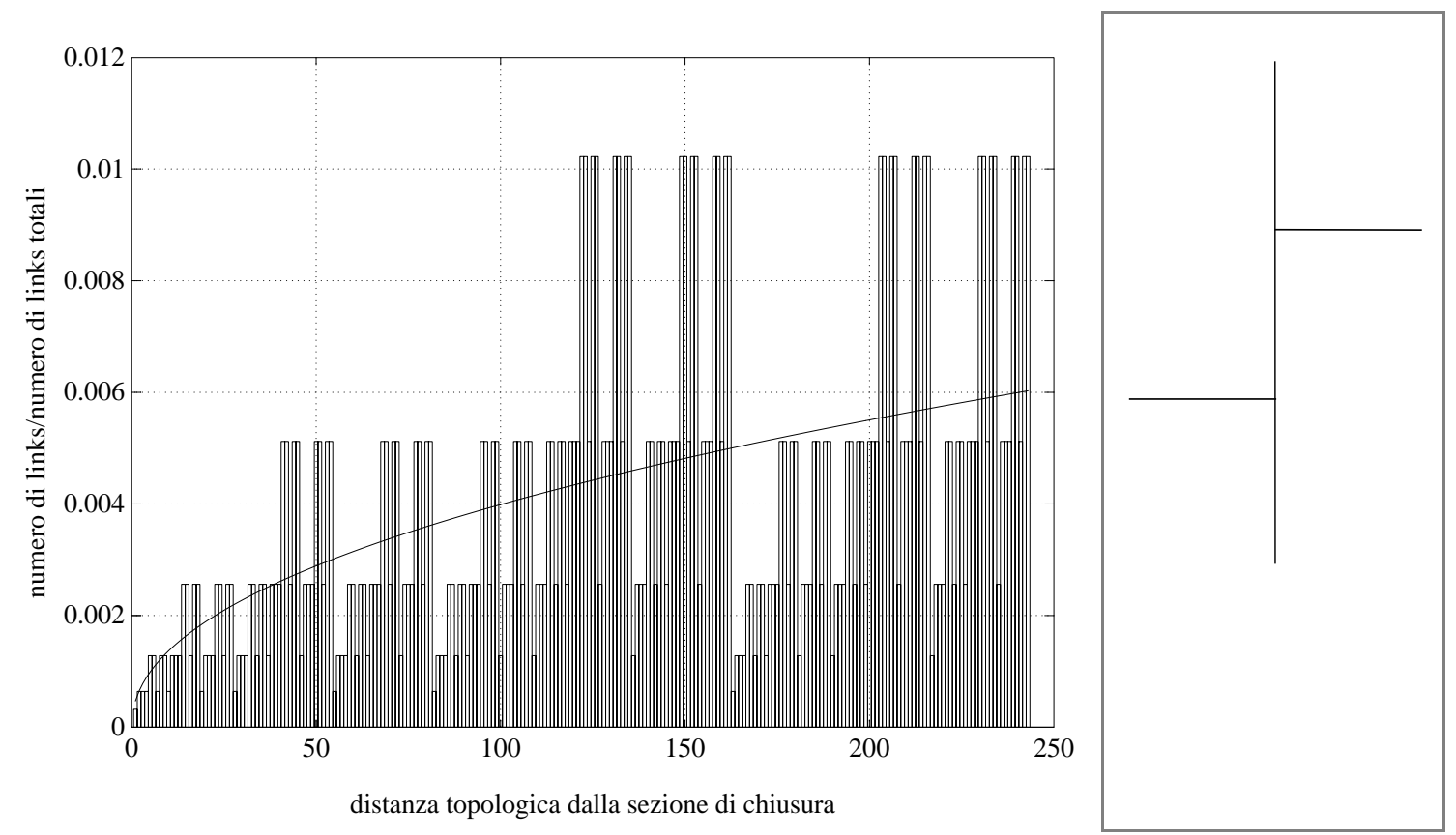

Fig. 4. Topological width function of the fractal network generated from the elementary tree represented in the rectangle on the right. The curve depicts the approximation represented by relation (20).

\section{THE MOST PROBABLE HYDROLOGIC RESPONSE}

The most probable hydrologic response of fractal networks can be found invoking the principle of maximum entropy (Jaynes, 1957), with some constraints. The constraints we can add are not arbitrary and introduce in the IUH formulation peculiar characteristics of fractal networks.

The first (obvious) constraint is the one ensuring that the sum of probabilities is unity:

$$
\sum_{\mathrm{i}=1}^{\Delta} \mathrm{p}_{\mathrm{i}}=\sum_{\mathrm{i}=1}^{\Delta} \frac{\mathrm{n}_{\mathrm{i}}}{\mathrm{N}}=1
$$

The second one should take into account some peculiar (physical) aspects of the network such as, for instance, the total length. What is of interest in the expression of the constraint is how structural parameters of the network appear. The total length is considered as a constraint in the expression

$$
\sum_{i=1}^{\Delta} \frac{n_{i}}{N} \cdot t_{i}^{D_{t}} \propto Z
$$

In fact, expanding the above relation using the uniform velocity $\mathrm{v}$ one obtains 


$$
\begin{aligned}
E\left[t_{i}^{D_{t}}\right] & =E\left[\left(\frac{L_{i}}{v}\right)^{D_{t}}\right]=E\left[\zeta^{D_{t}}\left(\frac{\Delta_{i}}{v}\right)^{D_{t}}\right]=\sum_{i=1}^{\Delta} \frac{D_{t} \cdot \Delta_{i}^{D_{t}-1}}{M} \cdot \zeta^{D_{t}}\left(\frac{\Delta_{i}}{v}\right)^{D_{t}}= \\
& =\sum_{\mathrm{i}=1}^{\Delta} \frac{\mathrm{D}_{\mathrm{t}} \cdot \zeta^{\mathrm{D}_{\mathrm{t}}}}{\mathrm{Mv}^{\mathrm{D}_{\mathrm{t}}}} \cdot \Delta_{\mathrm{i}}^{\mathrm{D}_{\mathrm{t}}-1} \cdot \Delta_{\mathrm{i}}^{\mathrm{D}_{\mathrm{t}}} \approx \frac{\mathrm{D}_{\mathrm{t}} \cdot \zeta^{\mathrm{D}_{\mathrm{t}}}}{\mathrm{Mv}_{\mathrm{t}}^{\mathrm{D}_{\mathrm{t}}}} \cdot \frac{\Delta^{2 \mathrm{D}_{\mathrm{t}}}}{2 \mathrm{D}_{\mathrm{t}}}= \\
& =\frac{\zeta^{\mathrm{D}_{\mathrm{t}}}}{\mathrm{v}^{\mathrm{D}_{\mathrm{t}}}} \cdot \frac{1}{2} \cdot \frac{\Delta^{2 \mathrm{D}_{\mathrm{t}}}}{\Delta^{\mathrm{D}_{\mathrm{t}}}}=\frac{\zeta^{\mathrm{D}_{\mathrm{t}}}}{\mathrm{v}^{\mathrm{D}_{\mathrm{t}}}} \cdot \frac{1}{2} \cdot \Delta^{\mathrm{D}_{\mathrm{t}}}=\frac{1}{2 \cdot \mathrm{v}^{\mathrm{D}_{\mathrm{t}}}} \cdot \mathrm{L}^{\mathrm{D}_{\mathrm{t}}}
\end{aligned}
$$

and considering (12) and (16) and taking into account that $\mathrm{L}_{\mathrm{i}}=\Delta_{\mathrm{i}} \zeta$, one can write:

$$
E\left[t_{i}^{D_{t}}\right]=\frac{1}{2 \cdot v^{D_{t}}} \cdot Z \Rightarrow E\left[t_{i}^{D_{t}}\right] \propto Z
$$

Considering $\mathrm{Z}$ as a constraint means that all information given by $\mathrm{Z}$ is incorporated into the expression of the most probable response, i.e. we will derive the most probable IUH consistent with the information given by $\mathrm{Z}$.

The second member of (25) is equivalent to the constant $\mathrm{k}$ in Lienhard and Meyer (1967):

$$
k=\frac{\mathrm{Z}}{2 \cdot \mathrm{v}^{\mathrm{D}_{\mathrm{t}}}}
$$

The third constraint is represented by relation (20). We have now relations (20), (22) and (25) as constraints equivalent to these used by Lienhard and Meyer (1967), with $\alpha=\beta=\mathrm{D}_{\mathrm{t}}$ resulting by construction for fractal networks. Equivalent constraints and equivalent framework produce the same IUH form as Lienhard and Meyer (1967). Therefore, according to (8), the most probable IUH is

$$
f(t)=\frac{D_{t}}{k} t^{D_{t}-1} \exp \left(-\frac{t^{D_{t}}}{k}\right)
$$

that, substituting $k$, becomes

$$
f(t)=D_{t} \frac{2 v^{D_{t}}}{Z} t^{D_{t}-1} \exp \left(-\frac{2 v^{D_{t}}}{Z} t^{D_{t}}\right)
$$

\subsection{Comparison with previous related works}

Troutman and Karlinger (1985), using the topological framework developed by Shreve $(1966,1967)$, derived the exact and asymptotic (for large n) IUH using different dynamic equations to characterize the residence time distribution of the water particles. For all dynamic equations, the expected IUH constrained to the knowledge of magnitude, is a Weibull function, depending only on $n$, on the average link length $l$, and on the celerity $c$ :

$$
f(t)=\left(\frac{\lambda^{2} t}{2 n}\right) \exp \left[-\frac{\lambda^{2} t^{2}}{4 n}\right]
$$


with $\lambda=c / l,{ }^{-}$. Parameters of expressions (29) and (28) of the Weibull distribution are related by:

$$
\frac{4 n}{\lambda^{2}}=\frac{\mathrm{Z}}{2 \cdot \mathrm{v}^{\mathrm{D}_{\mathrm{t}}}} ; \quad \mathrm{D}_{\mathrm{t}}=2
$$

In the comparison it is also assumed $\zeta=l,-, \mathrm{v}=c$ and $\mathrm{M}=2 n$.

Distribution (28) is plotted in figures 5 and 6 for $D_{t}$ varying between 1 and 2 , using $\zeta=1$ (so that $\mathrm{Z}=\mathrm{M}$ ) and $\mathrm{v}=1$. In figure $5, \mathrm{Z}$ is fixed at 200 and the curves obtained for different $\mathrm{D}_{\mathrm{t}}$ are compared with that arising from (29), with $c=1$ and $l,-=1$. We are using both $c$ and $\mathrm{v}$ with the same meaning of parameters converting length to time. In figure 5 it can be seen that the Troutman and Karlinger IUH has a peak that corresponds to that of the case $\mathrm{D}_{\mathrm{t}}=1.6$ but a greater average travel time. Comparison of figures 5 and 6 shows the difference in the effects of variation of $D_{t}$ with fixed $M$ and fixed $\Delta$. In the first case (figure 5) the increase of $D_{t}$, with $\mathrm{Z}$ fixed, implies a decrease of $\Delta$, according to (16), and a corresponding decrease of the average travel time with an increased peak. On the other hand, if $\Delta$ is fixed (figure 6), the increase of $D_{t}$ corresponds to a considerable expansion of the magnitude but to a relatively small increase of $\mathrm{E}[\mathrm{t}]$. Table 1 allows us to compare magnitude and topological diameters producing the IUH curves in both figures.

Looking at the mean travel times reported in table 1 it can be clearly recognized that in a fractal framework the key parameter for the determination of the value of $E[t]$ is the topological diameter. Extending this result to real world networks, the main stream length (and of course the average velocity) turns out to be the controlling factor for $E[t]$. Thus the fractal framework can give an explanation to the empirical evidence of the role of area or magnitude in the hydrologic response, since both variables could be related to the mainstream length through relations of the type of (16).

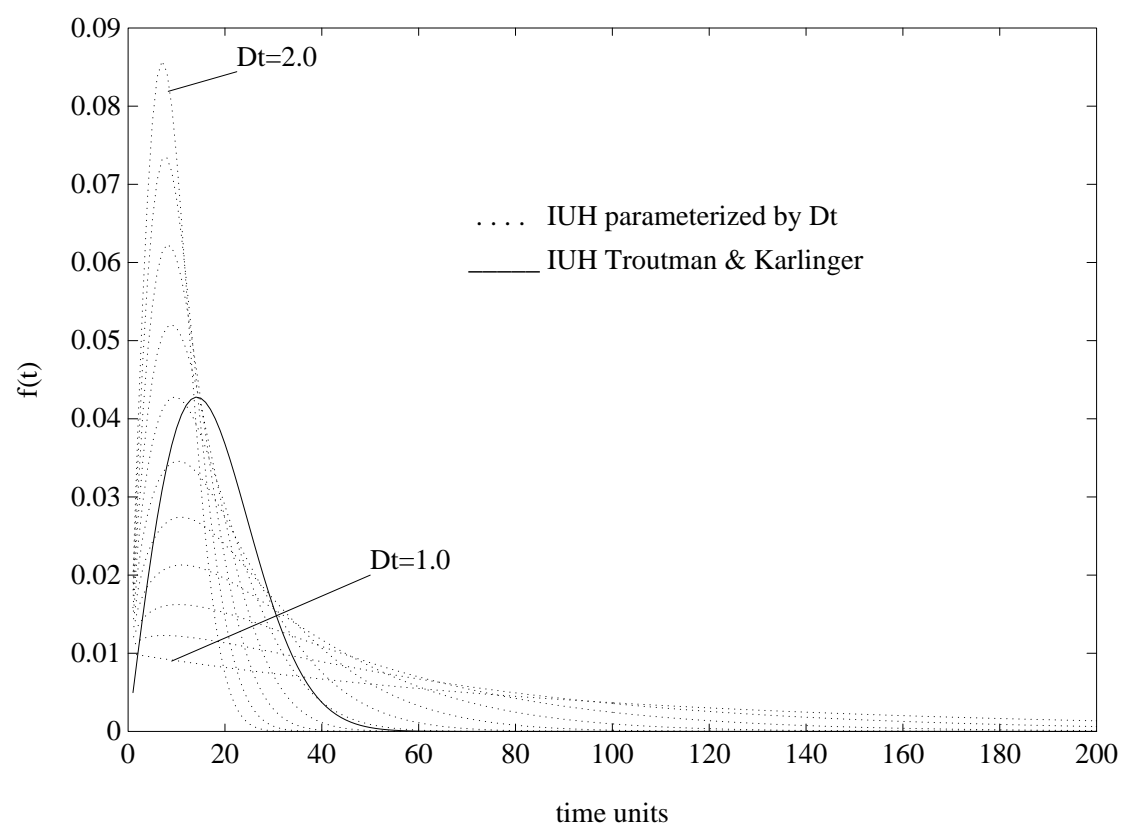

Fig. 5. Forms of the IUH of fractal networks for fractal dimension $D_{t}$ varying from 1.0 to 2.0, step 0.1. The segment length and the velocities are taken as unit values. Total length equals magnitude and both are fixed to $Z=M=200$. Due to relation (16), the topological diameter of the network decreases with increasing $D_{t}$. 


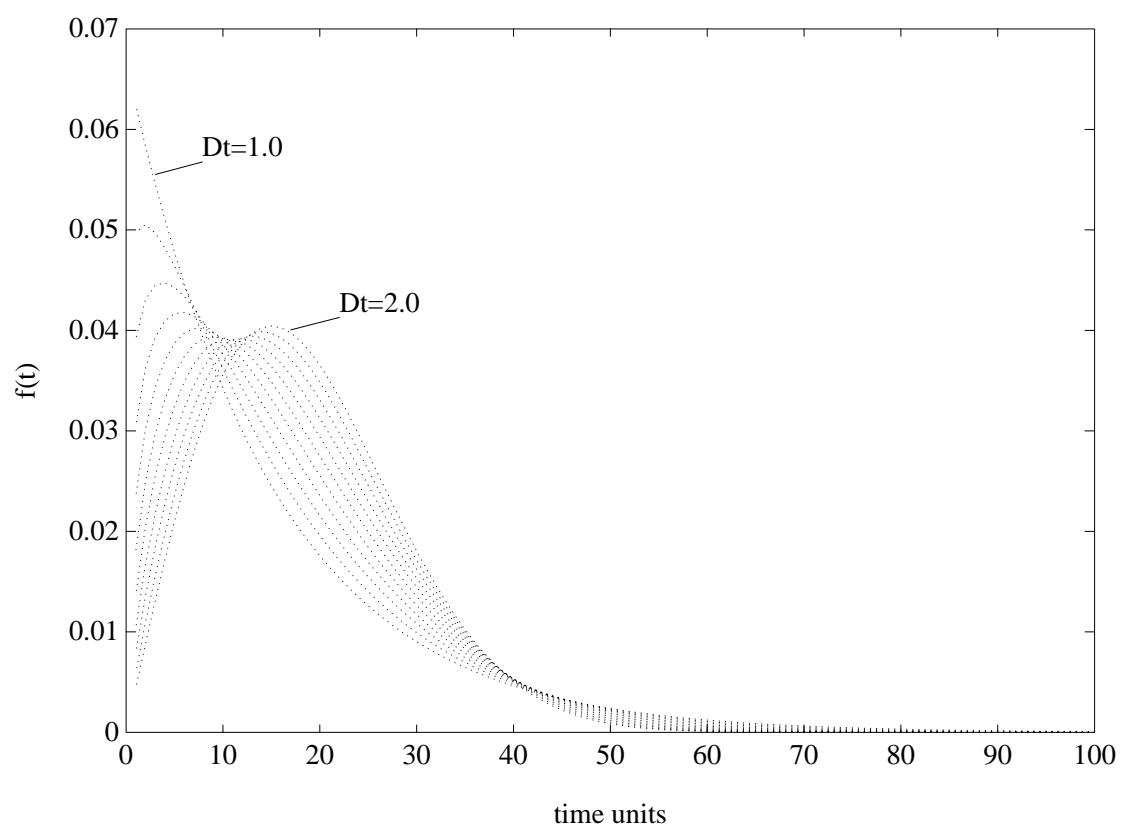

Fig. 6. Forms of the IUH of fractal networks for fractal dimension $D_{t}$ varying from 1.0 to 2.0, step 0.1. The segment length and the velocities are taken as unit values. The topological diameter is set as $\Delta=30$. Due to relation (16), total length and magnitude increase with increasing $D_{t}$.

\subsection{Moments of the IUH of fractal networks}

The first moment of distribution (8) is:

$$
E[t]=k^{1 / \alpha} \Gamma\left(1+\frac{1}{\alpha}\right)
$$

and can be expressed as a function of parameters of the impulse response of fractal networks:

$$
E[t]=\frac{1}{v}\left(\frac{Z}{2}\right)^{1 / D_{t}} \Gamma\left(1+\frac{1}{D_{t}}\right)
$$

and of the Troutman and Karlinger IUH parameters:

$$
E[t]=\left(\frac{4 n}{\lambda^{2}}\right)^{1 / 2} \Gamma\left(1+\frac{1}{2}\right)=\frac{\sqrt{\pi n}}{\lambda}
$$

where $\alpha$ is fixed to 2 . To compare these average travel times we have plotted (32) against $D_{t}$ for different values of $\mathrm{Z}$. Assuming $\zeta=1, \mathrm{Z}$ equals $\mathrm{M}$ and the comparison is meaningful. To provide a significant comparison between the mean travel times of the Troutman and Karlinger IUH and of the IUH of fractal networks we have plotted their ratio for different values of $Z$ and with $D_{t}$ varying from 1 to 2 . figure 7 shows that increasing the magnitude this ratio tends to attain unity in correspondence of $\mathrm{D}_{\mathrm{t}} \approx 1.65$, which is compatible with the 
position of the curves represented in figure 5. It seems also evident that for diverging magnitude the fractal dimension that ensure equality of the first moments does not exceed 1.7.

\begin{tabular}{|l|l|l|l|l|l|l|l|l|l|l|l|}
\hline $\mathrm{D}_{f}$ & 1 & 1.1 & 1.2 & 1.3 & 1.4 & 1.5 & 1.6 & 1.7 & 1.8 & 1.9 & 2.0 \\
\hline & & & & & & & & & & & \\
\hline$\Delta(M=200)$ & 200 & 124 & 83 & 59 & 44 & 34 & 27 & 23 & 19 & 16 & 14 \\
\hline $\mathrm{E}[\mathrm{t}]$ & 100.0 & 63.48 & 43.66 & 31.91 & 24.45 & 19.45 & 15.94 & 13.40 & 11.49 & 10.02 & 8.86 \\
\hline & & & & & & & & & & & \\
\hline $\mathrm{M}(\Delta=30)$ & 30 & 42 & 59 & 83 & 117 & 164 & 231 & 324 & 456 & 641 & 900 \\
\hline $\mathrm{E}[\mathrm{t}]$ & 15.00 & 15.42 & 15.84 & 16.26 & 16.67 & 17.06 & 17.44 & 17.80 & 18.15 & 18.48 & 18.80 \\
\hline
\end{tabular}

Tab. 1. Mean travel times E[t], with $D_{t}$ varying, in the two cases of $M$ and $\Delta$ fixed. Topological diameters $\Delta$ for fixed $M$ and total number of links $M$ for fixed $\Delta$ are also indicated.

An additional comparison can be made with the first moment of the IUH derived by Marani et al. (1991) for the Peano network, whose characteristics are described in the mentioned paper. The above authors derive $\mathrm{E}[\mathrm{t}]$ as

$$
E[t]=\frac{3}{2} \frac{L(\Omega, \Omega)}{v}
$$

that can be compared to the expression (33), considering that $\mathrm{L}(\Omega, \Omega)$ is the length of the stream of order $\Omega$ in the a Peano network of order $\Omega$. Given that, since Marani et al. (1991) assume for this plane-filling curve $\left(D_{t}=2\right)$ :

$$
\mathrm{L}(\Omega, \Omega)=\mathrm{R}_{\mathrm{B}}^{(\Omega-1) / 2} \varepsilon(\Omega),
$$

with $\varepsilon(\Omega)$ as the ruler, and given $n=\mathrm{R}_{\mathrm{B}}{ }^{\Omega-1}$, from the Horton law of stream numbers, setting $\lambda=\varepsilon(\Omega) / \mathrm{v}$ as assumed previously for fractal objects, makes (34) as

$$
\mathrm{E}[\mathrm{t}]=\frac{3}{2} \frac{1}{\sqrt{\pi}} \frac{\sqrt{\pi \mathrm{n}}}{\lambda}=0.846 \frac{\sqrt{\pi \mathrm{n}}}{\lambda}
$$

This allows us to use the curves reported in figure 7 for a comparison with this mean travel time, by making reference to the ordinate 0.846 of the diagram. 


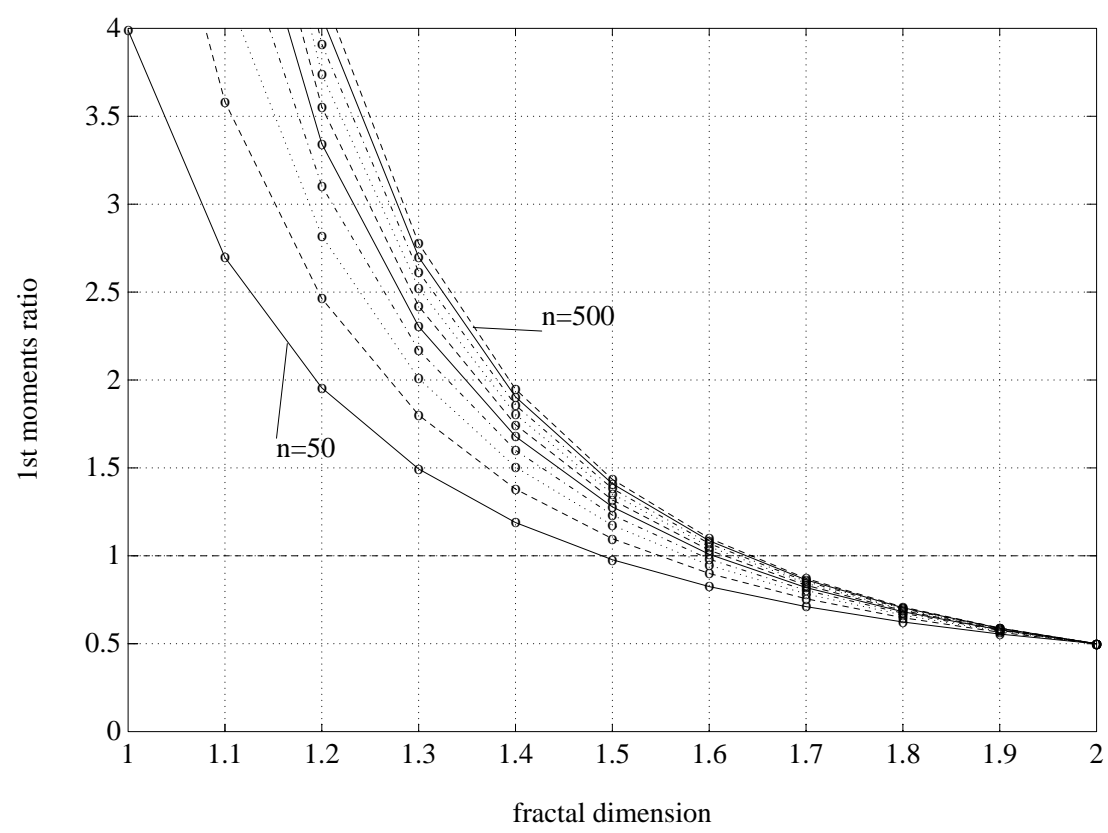

Fig. 7. Ratio $E_{F}[t] / E_{T K}[t]$ of the first moment of the travel time distribution for fractal networks $\left(E_{F}[t]\right)$ and for the scheme used by Troutman and Karlinger $\left(E_{T K}[t]\right)$. It is assumed $\zeta=1$ and $c=v=1$. Magnitude $n$ varies between 50 and 500 with step 50.

The second moment of the Weibull distribution (8) with respect to the origin is

$$
M_{2}(t)=k^{2 / \alpha} \Gamma\left(1+\frac{2}{D_{t}}\right)
$$

leading, in the case of fractal network, to

$$
M_{2}[t]=\frac{1}{v^{2}}\left(\frac{Z}{2}\right)^{2 / D_{t}} \Gamma\left(1+\frac{2}{D_{t}}\right)
$$

Computing values assumed by (37) for the same set of parameters used in table 1, the results reported in table 2 show that, when the topological diameter is fixed, there is a minimum of (37) for $D_{t}=1.4 \div 1.5$ while $M_{2}$ monotonically decreases with increasing $D_{t}$ when the magnitude is fixed. It is worth remarking that values of $D_{t}$ minimizing the second moment produce IUH with low peaks (figure 7). Moreover they in the range of topological fractal dimensions $\mathrm{D}_{\mathrm{t}}$ of the branching estimated by Claps and Oliveto (1993) on some river basins in Southern Italy using the informational entropy of the network (Fiorentino and Claps, 1992). 


\begin{tabular}{|l|l|l|l|l|l|l|l|l|l|l|l|}
\hline $\mathrm{D}_{\mathrm{t}}$ & 1 & 1.1 & 1.2 & 1.3 & 1.4 & 1.5 & 1.6 & 1.7 & 1.8 & 1.9 & 2.0 \\
\hline & & & & & & & & & & & \\
\hline$\Delta(M=200)$ & 200 & 124 & 83 & 59 & 44 & 34 & 27 & 23 & 19 & 16 & 14 \\
\hline $\mathrm{M}_{2}[\mathrm{t}]$ & 2000 & 7369 & 3242 & 1631 & 911 & 553 & 358 & 245 & 176 & 130 & 100 \\
\hline & & & & & & & & & & & \\
\hline $\mathrm{M}(\Delta=30)$ & 30 & 42 & 59 & 83 & 117 & 164 & 231 & 324 & 456 & 641 & 900 \\
\hline $\mathrm{M}_{2}[\mathrm{t}]$ & 450.0 & 434.5 & 426.5 & 423.3 & 423.2 & 425.3 & 428.7 & 433.2 & 438.4 & 444.0 & 450.0 \\
\hline
\end{tabular}

Tab. 2. Second moment $M_{2}[t]$ with respect to the origin, with $D_{t}$ varying, in the two cases of $M$ and $\Delta$ fixed.

\section{CONCLUSIONS}

A Weibull distribution with clearly defined parameters is obtained as the travel time distribution of a mass injected instantaneously in a fractal network. The maximization of the joint probability defining the arrival time distribution is made under three constraints which incorporate properties of fractal networks. This constitutes a step forward with respect to previous works in this field (e.g. Lienhard, 1964; Lienhard and Meyer, 1967; Marani et al., 1991) where the definition of constraints is either arbitrary or incomplete. Parameters driving the hydrologic response of fractal networks are the total network length and the fractal dimension, explicitly appearing in the arrival time distribution function.

The first moment of the distribution depends fundamentally on the network topological diameter. Translated in terms of real world rivers this result substantiates the significance of the mainstream length in the estimation methods to the basin lag time.

The influence of the fractal dimension is evident both in the peak discharge and in the second moment with respect to the origin, the latter presenting minima for $D_{t}=1.4 \div 1.5$. These values are of particular interest based on the results of an analysis of the fractal structure of river networks which was carried out by Claps and Oliveto (1993). 


\section{REFERENCES}

Claps, P and G. Oliveto, Fractal structure, entropy and energy dissipation in river networks, Hydrology jour. of the IAH, Vol. XVII, no. 3\&4, 38-51, 1994.

Feder J. (1988) Fractals, Plenum Press, New York.

Fiorentino, M. e P. Claps, On what can be explained by the entropy of a channel network, in Singh e Fiorentino (Eds.) Entropy and Energy Dissipation in Water Resources, 139-154, Kluwer, Dordrecht, The Netherlands, 1992.

Fiorentino M., P.Claps e V.P.Singh, An entropy-based morphological analysis of river-basin networks , Water Resour. Res.,29(4), 1215-1224, 1993.

Gupta V. M. and E. Waymire. On the formulation of an analitycal approach to hydrologic response and similary at basin scale, Journal of Hydrology, 65, 95-123, 1983.

Jaynes, E. T., Information theory and statistical mechanics, I. Phys. Rev., 106, 620-630, 1957.

La Barbera, P. and R. Rosso, On the fractal dimension of stream networks, Water Resour. Res., 25(4), 735-741, 1989.

Lienhard, J. H., A Statistical mechanical prediction of the dimensionless unit hydrograph. $J$. Geophys. Res., Vol. 69, N.24, 5231-5238, 1964.

Lienhard, J. H. and P.L. Meyer, A physical basis for the generalized Gamma distribution, Quart. Appl. Math., 25, 330-334, 1967.

Mandelbrot, B.B., The Fractal Geometry of Nature, W.H. Freeman, New York, 1983.

Marani, A., R.Rigon and A. Rinaldo, A note on fractal channel networks, Water Resour. Res., 27 (12), 3041-3049, 1991.

Rinaldo, A., Marani, A. e R.Rigon, Geomorphological dispersion, Water Resour. Res., 27 (4), 513-525,1991.

Rinaldo, A., Rodriguez-Iturbe I., R.Rigon, R.L.Bras, E.Ijjasz-Vasquez and A.Marani, Minimum Energy and fractal structures of drainage networks, Water Resour. Res., 28 (9), 2183-2195,1992.

Shreve R. L. Statistical law of stream numbers, J. Geology, vol.74, pp. 17-37, 1966.

Shreve R. L. Infinite topologically random channel network, J. Geology, vol.75, pp. 179-186, 1967.

Troutman B. M. and M. B. Karlinger. Unit hydrograph approximation assuming linear flow trough topologically random channel networks, Water Resour. Res., 21(5), 743-754, 1985.

\section{Acknowledgments}

This work was supported by funds granted by the Italian Ministero della Ricerca Scientifica $e$ Tecnologica, Progetto 40\% "Processi Idrologici Fondamentali". 